\title{
Der Informationsgehalt von Indikatoren des Technologietransfers in peripheren Regionen
}

\author{
Caron Pomp $\cdot$ Stefan Zundel
}

Online publiziert: 28. Juli 2020

(C) Der/die Autor(en) 2020

Zusammenfassung An Hochschulen wird vermehrt die Erwartung herangetragen, einen Beitrag zu regionalen Wirtschaftsentwicklung zu leisten. In diesem Kontext, der oft als Teil der so genannten Third Mission angesehen wird, wird auch gefordert, Output und Wirkung des Technologietransfers auf die regionale Wirtschaft durch Indikatoren möglichst gut zu erfassen. Eine Literaturauswertung liefert eine Liste jener Indikatoren, die in der Literatur zu diesem Zweck häufig angeführt werden. Diese Indikatoren können den Erfolg von Transferleistungen aber nur beschränkt abbilden, weil es eine Vielzahl von intermittierenden Faktoren gibt, die die Wirkung einer Hochschule auf die sie umgebende regionale Wirtschaft beeinflussen. Darüber hinaus muss eine schwache Performance von Hochschulen im Technologietransfer in peripheren Regionen nicht die mangelnde Leistungsfähigkeit einer dieser Hochschule signalisieren. Dafür können auch strukturelle Bindungsprobleme zur umgebenden Region und die fehlende Absorptionsfähigkeit der regionalen Wirtschaft verantwortlich sein.

Schlüsselwörter Technologietransfer · Wirkungsmessung · Indikatoren · Periphere Regionen

C. Pomp $(\bowtie) \cdot$ S. Zundel

Fachgebiet Allgemeine VWL mit dem Schwerpunkt Energie- und Umweltökonomik, Brandenburgische Technische Universität Cottbus-Senftenberg, Senftenberg, Deutschland E-Mail: robertpomp@gmail.com 


\section{The information content of transfer indicators in peripheral regions}

Abstract Universities are increasingly expected to contribute to regional economic development. In this context, which is often regarded as part of the so-called third mission, there is also a demand to evaluate the technology transfer outputs and their impact on the regional economy as precise as possible. A literature review provides a list of those indicators that are frequently cited in the literature for this purpose. However, these indicators can only reveal the success of transfer services to a limited extent because there is a multitude of intermittent factors that influence the impact of a university on the surrounding regional economy. In addition, a poor transfer performance by universities in peripheral regions does not necessarily signal the lack of the overall performance of these universities. This can also be due to structural matching problems with the surrounding region and the lack of absorption capacity of the regional economy.

Keywords Technology transfer · Impact measurement · Indicators · Peripheral regions

\section{Einleitung}

Seit dem Lissabon-Gipfel der EU im Jahr 2000 gilt es als politischer Konsens, dass die Innovationsstätigkeit ein zentraler Treiber der wirtschaftlichen Entwicklung ist. Dementsprechend werden Hochschulen neben ihren traditionellen Aufgaben in Forschung und Lehre zunehmend auch in den Dienst der Wirtschaftsentwicklung der sie umgebenden Region genommen (EU 2011). Die strategische Fokussierung auf Innovationen spiegelt sich auch in den nationalen Strategien der Mitgliedsstaaten der EU wider. Die High-Tech-Strategie 2025 der Bundesregierung ist ein Beispiel dafür (Bundesregierung 2018). Im Fokus der Länderpolitik steht stärker die regionale Wirkung von Hochschulen. Ein aktuelles Beispiel für diese politische Orientierung ist die Förderung der Hochschulen in der Lausitz als Reaktion auf den geplanten Ausstieg aus der Braunkohleverstromung (MWFK 2019a, b). Neben der Ausbildungsleistung und den Wertschöpfungseffekten, die durch die Nachfrage nach Gütern und Dienstleistungen durch Universitäten und ihrer Angehörigen ausgelöst werden können, stehen vor allem die Wirkung der Hochschulen auf die Innovationstätigkeit der Regionen im Mittelpunkt des politischen Interesses.

Konzeptionell spiegelt sich diese Erwartung in dem Wunsch nach einem veränderten Rollenverständnis von Hochschulen wider. Unter dem Begriff der „Triple Helix University“ werden vor allem Hochschulen angesprochen, die aktive Bestandteile der regionalen Innovationsnetzwerke sind (Etzkowitz 2008). Der Begriff der ,Entrepreneurial University“ fokussiert demgegenüber stärker auf die Wirkung auf das Gründungsgeschehen allgemein und in der umgebenden Region (Etzkowitz 1997). Der Wissenschaftsrat macht sich ebenfalls für eine Stärkung des Wissens und Technologietransfers (,third mission“) stark und fordert eine Strategieentwicklung in den Hochschulen, die der Bedeutung dieses Tätigkeitsbereiches angemessen ist (Wissenschaftsrat 2016). 
In die Förderung der Wissenschaft allgemein und die der Hochschulen fließen deshalb erhebliche Mittel. Im Jahr 2015 flossen im Schnitt über 650.000€ Grundmittel und etwa 300.000€ Drittmittel je Professorin und Professor an die deutschen Hochschulen (Statistisches Bundesamt 2018). Allein die Förderinitiative „Innovative Hochschule“ stellt bis 2027 bis zu 550 Mio. $€$ zur Stärkung der Dritten Mission bereit ${ }^{1}$. Die Mittelgeber möchten ebenso wie die Mittelempfänger wissen, ob und in welchem Umfang eine Wirkung zu sehen ist. Das Interesse richtet sich insbesondere auf den Technologietransfer, eine Domäne der Ingenieur- und Naturwissenschaften; und es richtet sich auf die regionale Wirkung, weil von den Hochschulen und Forschungseinrichtungen ein Einfluss auf die Nivellierung regionaler wirtschaftlicher Disparitäten erwartet wird (Brachert et al. 2019). Es verwundert deshalb kaum, dass die Hochschulen ebenso wie die staatlichen Geldgeber ein großes Bedürfnis haben, die Wirkung von Hochschulen auf die Entwicklung der sie umgebenden regionalen Wirtschaft zu messen.

An Indikatoren für den Technologietransfer und seiner Wirkung ist kein Mangel, wie noch $\mathrm{zu}$ zeigen sein wird (siehe Abschn. 3). Aber leisten diese Indikatoren das, was sie leisten sollen? Zeigen sie einen Effekt und vor allem, stellen sie den gewünschten Zusammenhang zwischen der Messung des Technologietransfers einer Hochschule und einer Wirkung auf die Wirtschaft, insbesondere auf die regionale Wirtschaft, her?

Im Folgenden soll gezeigt werden, dass die gebräuchlichsten Indikatoren nur in sehr engen Grenzen den Erfolg von Transferleistungen abbilden können, weil es eine Vielzahl von intermittierenden Faktoren gibt, die die Wirkung einer Hochschule auf die sie umgebende regionale Wirtschaft beeinflussen. Diese Faktoren, die mit der Leistung einer Hochschule wenig zu tun haben, können die Wirkung einer Hochschule auf die Umgebung erheblich beeinflussen und stören. Darüber hinaus führt der Vergleich von Hochschulen und Forschungseinrichtungen in metropolitanen Regionen mit solchen Einrichtungen in peripheren und strukturschwachen Regionen in die Irre. Die Performance von Hochschulen in peripheren Regionen signalisiert weniger mangelnde Leistungsfähigkeit, sondern eher strukturelle Bindungsprobleme zur umgebenden Region und die fehlende Absorptionsfähigkeit der regionalen Wirtschaft.

Zur Begründung dieser beiden Thesen gehen wir wie folgt vor: In Abschnitt zwei resümieren wir den theoretischen Hintergrund der Erfolgsmessung des Technologietransfers und stellen bedeutsame empirische Befunde zum Verhältnis von Hochschulen/Forschungseinrichtungen zur Umgebungsregion vor, die die Aussagekraft von Indikatoren des Technologietransfers beeinflussen. In Abschnitt drei präsentieren wir das Ergebnis einer Literaturauswertung zu den gebräuchlichsten Indikatoren und erläutern deren Bedeutung. Im vierten Abschnitt erfolgt eine Beurteilung dieser Indikatoren vor dem Hintergrund des aktuellen Standes der Forschung zum Zusammenhang zwischen Hochschulen und Wirtschaft. Der fünfte und abschließende Abschnitt beinhaltet eine Zusammenfassung der Ergebnisse und einen Ausblick auf eine mögliche Weiterentwicklung der Indikatorik für periphere Räume.

\footnotetext{
1 https://www.innovative-hochschule.de/de/foerderinitiative.
} 


\section{Der theoretische Hintergrund und vorhandene empirische Befunde}

Der Begriff des Wissenstransfers (WT) umfasst Systeme und Prozesse, bei denen Wissen, Expertise und ausgebildete Personen zwischen einer wissenschaftlichen Institution (Universitäten und Forschungseinrichtungen) und Nutzergruppen aus den Bereichen Wirtschaft, Staat, Öffentlichkeit und der Zivilgesellschaft ausgetauscht werden (Research Councils UK 2007; Rossi und Rosli 2015). Der Technologietransfer (TT) ist jener Teil des Wissenstransfers, der auf anwendbares Wissen aus den Natur- und Ingenieurwissenschaften, der Medizin sowie der Mathematik zielt. Wissenstransfer ist gegenüber dem Technologietransfer der allgemeinere Begriff und kann auch die Anwendung von Wissen aus den Geistes- und den Sozialwissenschaften umfassen. Wissenstransfer muss auch nicht zwangsläufig auf die Anwendung von Wissen zielen, sondern kann auch einfach Bildung und Unterhaltung sein, beispielsweise über offene Formate wie die Lange Nacht der Wissenschaft (Schulz 2013, S. 29-30).

Die Definition des TT ist keineswegs so eindeutig wie es scheint. Eine Technologie bzw. das Wissen um sie ist keine Entität, die von A nach B wechselt wie ein Geldschein den Besitzer. Oft ist es schwierig abzugrenzen, wo eine Technologie aufhört und eine andere anfängt (Bozemann 2000). Der Prozess des Technologietransfers kann über viele verschiedene Kanäle ablaufen (Teixeira et al. 2020; Schubert et al. 2012); und vor allem ist das Bild, wonach ein Anbieter von Wissen einem Nachfrager von Wissen etwas übereignet, schief. Oft handelt es sich um eine Koproduktion zwischen Wissenschaftlern und Anwendern, bei der ein bidirektionaler Austausch von Wissen praktiziert wird (Rossi und Rosli 2015, S. 1972).

Der TT hat in den letzten 20 Jahren in Deutschland und nicht nur hier eine steile politische Karriere absolviert. Die Basis für dieses Erfolgsmodell ist der empirische Befund, wonach das Wachstum von Volkswirtschaften positiv mit den Ausgaben in FuE und für die Hochschulen korreliert (Aghion und Howitt 2006). Allerdings zeigte sich in ihrer Untersuchung auch, dass dieser Effekt umso stärker ausfällt, je mehr die Firmen dieser Volkswirtschaften am aktuellen technologischen Entwicklungsrand operieren. Anders ausgedrückt: Ökonomien, deren Firmen überwiegend nicht im High Tech Bereich angesiedelt sind, können den Produktionsfaktor Wissen deutlich weniger nutzen. Diese empirischen Befunde ordnen sich ein in eine Wachstumstheorie, die sich in die Schumpetersche Tradition stellt, und die schöpferische Zerstörung durch neues Wissen betont. Neues Wissen ist in dieser Sicht der entscheidende Produktionsfaktor (Aghion et al. 1998).

Das kausale Modell, das der hier diskutierten Indikatorik zugrunde liegt, kann auf diese Wachstumstheorie zurückgeführt werden. Idealtypisch und sehr vereinfacht lässt sich der Zusammenhang wie folgt ausdrücken: Ausgaben für die Verbesserung von Hochschulen und Forschungseinrichtungen erhöhen deren Output, insbesondere in Form von qualifizierten Absolventen und neuen Forschungsresultaten. Dieser Output verbessert wiederum die Ressourcenausstattung des ökonomischen (Umgebungs-)Systems. Er kann umgewandelt werden in einen Input für das ökonomische System: das Humankapital ist besser qualifiziert und neues Wissen kann in neue Geschäftsmodelle umgesetzt werden. Am Ende erhöht dies den Output des ökonomischen Systems, weil neue Produkte entstehen und die Produktivität 


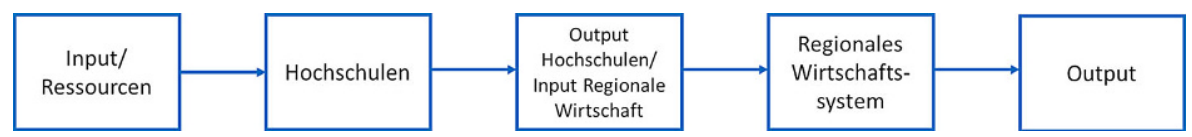

Abb. 1 Die Wirkung Hochschulen auf die Wirtschaft - eine idealtypische kausale Kette

erhöht wird. Die Abb. 1 zeigt schematisch diese kausale Kette beginnend mit den Hochschulen.

Die geschilderte kausale Kette ist keine Einbahnstraße; eine Reihe von Rückkopplungseffekten, die in der Zeichnung nicht abgebildet wurden, ist evident. Ein wohlhabendes Land kann mehr Ressourcen in das Wissenschaftssystem investieren. Die Unternehmen einer wirtschaftlich starken Region können eher die vielfach erforderliche Ko-Finanzierung bei Drittmittelprojekten aufbringen. Starke Unternehmen und ihre Forschungsabteilungen sind oft wichtige Partner, die gemeinsame Projekte mit einer Hochschule auch wissenschaftlich voranbringen können. Weil auch Unternehmen nach optimaler Passung und wissenschaftlicher Exzellenz suchen, ist keineswegs ausgemacht, dass die räumliche Nähe zu einer Hochschule auch eine Zusammenarbeit stiftet. Es lassen sich also auch Pfeile in die andere Richtung ziehen. In diesem Argumentationskontext soll der Fokus jedoch auf dem Gedanken liegen, dass die Verbindung zwischen Investitionen in das Wissenschaftssystem und einem wirtschaftlichen Effekt vielfach vermittelt ist.

Die empirische Evidenz für einen Zusammenhang zwischen FuE-Anstrengungen einerseits, beispielsweise gemessen in öffentlichen Ausgaben für FuE, und der Performance des ökonomischen Systems andererseits, ausgedrückt in Produktivität oder Wachstumsraten lässt sich kaum bestreiten. Ein Überblick zu den Befunden findet sich bei Schubert et al. (2012), bei Bonnacorsi (2017) und bei Teixeira et al. (2020). Allerdings gibt es zwischen einer Erhöhung der Ausgaben für Hochschulen und einer Wirkung, insbesondere auf die Wirtschaft der umgebenden Region, eine ganze Reihe von Einflussgrößen, die die Wirkung von Hochschulen auf die sie umgebende regionale Wirtschaft beeinflussen oder gar zunichtemachen können. Im Folgenden werden die wichtigsten Faktoren aufgeführt:

Gute Forschung muss eine kritische Masse aufweisen, um ökonomisch wirksam zu werden (Bonaccorsi et al. 2006). Diese ist erforderlich, um innerhalb der Forschungsteams Arbeitsteilung praktizieren zu können und durch Akteure aus der Wirtschaft als kompetente akademische Ansprechadresse für ein bestimmtes Forschungsfeld oder eine bestimmte Fragestellung wahrgenommen zu werden. Wird ein Schwellenwert für eine solche kritische Masse nicht erreicht, ist die ökonomische Wirkung singulärer Forscher deutlich reduziert. Allerdings gelten diese Befunde nur für die ökonomische Verwertung von Forschungsergebnissen, die forschungsgetrieben sind. Das kann sich bei problemgetriebenen Kooperationen zwischen Wissenschaft und Wirtschaft anders darstellen, weil diese i.d.R. ohnehin kleinteiliger sind.

Exzellente Forschung und wirtschaftliche Verwertbarkeit müssen sich nicht zwangsläufig ausschließen. Empirische Befunde deuten darauf hin, dass Spitzenforscher auch ökonomisch sehr erfolgreich sein können (Bonnacorsi 2017, S. 300 und die dort angegebene Literatur). Allerdings ist die Motivation der Akteure 
im Wissenschaftsbetrieb nicht primär ökonomisch, sondern reputationsorientiert (Kempton 2019). Kooperationen mit der Wirtschaft, die sich nicht oder nur schwer mit der Eigenlogik des Wissenschaftsbetriebes vereinbaren lassen, haben es deshalb schwerer. Forscher in den Herkunftsbereichen des TT sind primär an Kooperationen mit finanzstarken und/oder High-Tech-Unternehmen interessiert, die ihnen bei ihren wissenschaftlichen Aufgaben weiterhelfen können (Cooke 2001).

Um wirksam zu sein, müssen Hochschulen ein ähnliches Spezialisierungsmuster aufweisen wie die umgebende Wirtschaft (Boschma und Gianelle 2014). Es ist naheliegend, dass beispielsweise Forschung auf einem speziellen Forschungsfeld von der regionalen Wirtschaft nicht verarbeitet werden kann, wenn es keine Firmen in der regionalen Umgebung gibt, die sich auf ein verwandtes Geschäftsfeld spezialisiert haben. In diesem Fall suchen sich die Wissenschaftler anderenorts Partner. Das gilt auch umgekehrt: Firmen, die sich auf ein bestimmtes Geschäftsfeld spezialisiert haben und keine Pendant dazu an der regionalen Hochschule finden, suchen sich akademische Partner in anderen Regionen.

Das Wissen, das an Hochschulen produziert wird, liegt selten in einer Form vor, die von Firmen unmittelbar genutzt werden kann. Ereignis- oder objektbezogenes explizites Wissen kann als kodifiziertes Wissen in verschiedenen Formen dokumentiert, gespeichert, verarbeitet und dementsprechend weitergegeben werden (Schmidt 2013, S. 23-24). Ein persönlicher Kontakt ist bei dieser Art von Transfer nicht zwingend notwendig. Implizites Wissen oder ,tacit knowledge“ hingegen ist an Personen gebunden und kann nur über den persönlichen Kontakt weitergegeben werden. Daher spielen formelle wie auch informelle Interaktionen zwischen Mitarbeitern der Hochschulen und der Unternehmen über gemeinsame Forschungsprojekte ebenso eine wichtige Rolle.

Die wechselseitige Kenntnis von FuE-Möglichkeiten zwischen Forschung und Wirtschaft ist die Minimalvoraussetzung für die Etablierung eines gemeinsamen Projektes und sie kann wegen hoher Suchkosten nicht als selbstverständlich vorausgesetzt werden. Know How muss sodann in Prototypen übersetzt werden; diese müssen getestet werden; vielfach sind ein Upscaling und ein Prozessengeneering erforderlich. Für alle diese Prozesse bedarf es geeigneter Institutionen, die als Intermediäre zwischen Wissenschaft und Wirtschaft operieren. Räumliche Nähe von Wirtschaft und Wissenschaft allein ist deshalb kein Garant einer erfolgreichen wirtschaftlichen Verwertung von wissenschaftlichen Erkenntnissen (Boschma 2005, S. 63-64).

Ein weiterer wichtiger Einflussfaktor wird in der Literatur mit dem Begriff der $A b$ sorptionskapazität (Cohen und Levinthal 1990) der regionalen Wirtschaft adressiert. Allgemein ist damit die Fähigkeit von Firmen gemeint, Wissen, das an Hochschulen oder Forschungseinrichtungen produziert wird, in einen wirtschaftlich verwertbaren Anwendungszusammenhang zu übersetzen. Die Absorptionskapazität hängt vom Umfang der Ausgaben für Forschung in den Firmen (je mehr, desto besser) (Cohen und Levinthal 1990, S. 138), von der Nähe der Firmenaktivitäten zur Forschungsfront (je mehr High tech, desto besser, siehe oben) und von dem Vorhandensein geeigneter „Übersetzer“ in einer Firma (je mehr hoch qualifizierte Personen, desto besser) ab (Cohen und Levinthal 1990, S. 127). Auch die Firmengröße wird als Prädikator für die Absorptionskapazität herangezogen. Gesichert ist, dass KMU, insbesondere sehr kleine Unternehmen, im statistischen Durchschnitt weniger innovativ sind als 
größere Unternehmen (Kritikos et al. 2017). Umstritten ist hingegen, ob die Absorptionskapazität von neuem Wissen mit der Unternehmensgröße korreliert (Zou et al. 2018).

Im konzeptionellen Rahmen des entrepreneurial eco-system Ansatzes wird vor allem auf Unternehmensgründungen abgestellt, die für die Prosperität eine regionale Wirtschaft von essentieller Bedeutung sind (Stam 2015; Berger et al. 2018). Oft sind es Gründer, die Wissen aus einer Hochschule in Geschäftsmodelle übersetzen. In diesem Kontext werden zwei Einflussfaktoren besonders angesprochen: der Gründergeist, der entrepreneurial spirit oder die Gründerkultur, mit dem die Bereitschaft angesprochen wird sich selbstständig zu machen und ein unternehmerisches Risiko einzugehen (Fritsch und Wyrwich 2014) und das entrepreneurial ecosystem, mit dem alle Umfeldbedingungen angesprochen werden, die unternehmerisches Tun erleichtern oder erschweren können. Diese schließen die institutionellen Rahmenbedingungen unternehmerischen Tuns ebenso ein wie die kulturellen.

Kempton (2019) weist darauf hin, dass die Idee, wonach der TT sich positiv auf die regionale Wirtschaft der Umgebung einer Hochschule auswirkt, im Wesentlichen einer Handvoll instruktiver Beispiele abgeschaut ist: u. a. dem Silicon Valley, dem goldenen Dreieck Cambridge/Oxford/London oder dem MIT-Korridor an der Ostküste der Vereinigten Staaten. Die Wirkung des TT ist aber in peripheren Regionen, wo ein erfolgreicher TT besonders nötig wäre, eine andere und vielfach auch eine schwächere. Der TT hat, folgt man den Befunden in der Literatur, einen deutlichen regionalen Bias:

- Weil sie oft klein und fachlich in der Breite und nicht in der Spitze aufgestellt sind, können Hochschulen in der Provinz die kritische Masse nicht darstellen, die erforderlich ist, um attraktiv für die Wirtschaft zu sein (Bonnacosi 2017).

- Die Eigenlogik des Wissenschaftsbetriebes lässt gerade das Forschungspersonal an Universitäten vor einer Zusammenarbeit mit einer regionalen und kleinteiligen Wirtschaft zurückschrecken (Kempton 2019 und die dort angegebene Literatur).

- Die Absorptionsfähigkeit von peripheren Regionen ist deutlich geringer als in Wirtschaftszentren (Bonnacorsi 2017, S. 297). Die Unternehmen sind im Durchschnitt kleiner; der Umfang der FuE-Ausgaben ist geringer; der technologische Entwicklungsstand vieler regionaler Unternehmen ist oft middle und nicht high tech; und die Zahl der Personen, die in Unternehmen Übersetzerdienste bei der Umsetzung von Forschungsergebnissen leisten könnten, ist ebenfalls in peripheren Wirtschaftsräumen geringer.

- Der Gründergeist und das regionale Eco-System scheinen einen regionalen Bias aufzuweisen. Das gilt zumindest für die ländlichen Regionen der neuen Bundesländer, denen attestiert wird noch immer unter den Nachwehen der DDR zu leiden, in der Selbständigkeit diskriminiert wurde (Fritsch und Wyrwich 2014, S. 956).

- Hinzu kommt, dass Universitäten auf die Qualifikation der Beschäftigten in einer Region eine ambivalente Wirkung haben. Durch ihre Ausbildungsleistung können sie diese verbessern; sie können aber auch als Durchlauferhitzer für andere Arbeitsmärkte als den regionalen Arbeitsmarkt dienen und entziehen damit der Region qualifiziertes Personal, da insbesondere Hochschulabsolventen eine ho- 
he Mobilität aufweisen (Bonaccorsi 2017, S. 298; Faggian und McCann 2008, S. 324).

Das muss nicht heißen, dass TT in peripheren Wirtschaftsregionen nicht möglich ist und Hochschulen dort überhaupt keinen signifikanten Einfluss auf die regionale Wirtschaft haben (siehe hierzu Valero und van Reenen 2019), aber die Befunde deuten darauf hin, dass eine Reihe von Vorkehrungen getroffen werden müssen, um Transfer regional zu adaptieren und wirksamer zu machen. Die geschilderten Befunde haben vor allem Auswirkungen auf die Frage, was sich mit Indikatoren im Bereich des TT messen lässt und was nicht.

\section{3 Übersicht über vorhandene Indikatoren in der Literatur}

In der Literatur ist eine Vielzahl von Indikatoren im Gebrauch, die den quantitativen Erfolg des TT anzeigen sollen. Um einen aktuellen Überblick zu bekommen, wurde wie folgt vorgegangen.

Unter den Schlagworten „university“, „HEI“ (high education institution), ,technology“, „knowledge“, „transfer“, ,evaluation“ und ,,indicator“" wurden eine Reihe wissenschaftlicher Suchmaschinen für den Zeitraum 2000 bis 2019 auf einschlägige Veröffentlichungen untersucht. Dieser Zeitraum bot sich an, weil er in etwa mit dem Beginn der Lissabon-Strategie der EU korrespondiert. Die Suchmaschinen waren Sciencedirect/Elsevier, Jstor, Tandfonline, Wiley, Springer.Link, scholar.google. Der Fokus lag auf solchen Studien, die den TT mithilfe von Indikatoren messen oder eine TT-Indikatorik insgesamt bewerten.

Um die Indikatorik in eine Ordnung zu bringen, wurde die kausale Kette (siehe Abb. 1) genutzt. Abb. 2 setzt nun die Indikatoren in eine Beziehung zu den einzelnen Gliedern dieser Kette.

Der „Input“ beschreibt all jene Ressourcen, die Transferaktivitäten erst ermöglichen, wie Personal- oder Sachmittel (Rassehövel 2010, S. 84; Wissenschaftsrat 2016, S. 19). Der „Output“ bemisst die Produktivität, also das erzeugte und transferierte Wissen oder das unmittelbare Produkt einer Transferaktivität, wie etwa Publikationen, Erfindungen oder Ausstellungen (Rassehövel 2010, S. 84; Wissenschaftsrat 2016, S. 19; Woll 2011, S. 28). Als Outcome werden diejenigen Outputs bezeichnet, die Transferpartner durch Anschlusshandlungen an die Transferaktivität erzeugen, wie etwa neue Produkte oder Prozesse in einem Unternehmen oder auch Gründungen (Wissenschaftsrat 2016, S. 19). Am Ende der Kette steht der „Impact“, dieser umfasst die Nutzenstiftung der Transferaktivitäten und darauf begründeter Innovationen für Gesellschaft, Wirtschaft und Politik (Wissenschaftsrat 2016, S. 19).

Die Erfolgsmessung beruht auf dem Gedanken, dass sich zwischen den Indikatoren, die den Input oder den Output von Hochschulen ( $\sim \mathrm{HEI})$ messen, einerseits und den Indikatoren, die entweder die Relation zwischen Input und Output des regionalen Innovationssystems messen, oder aber den Output selbst, eine Beziehung herstellen lässt. Idealerweise müsste sich dann ein Zuwachs in ersterem Bereich auch in einem Zuwachs etwa bei der Produktivität oder dem Wachstum des Brut- 


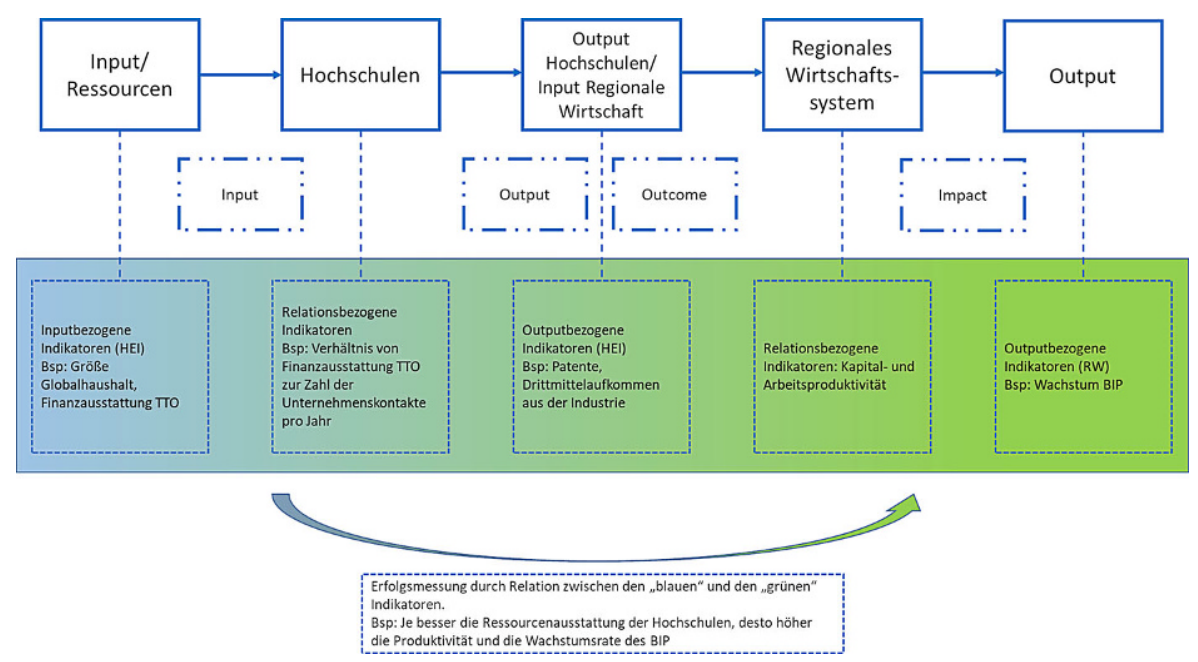

Abb. 2 Der Technologietransfer und seine Indikatoren

toinlandsproduktes niederschlagen, wenn der TT eine Wirkung auf das regionale Wirtschaftssystem haben soll.

Tab. 1 gibt einen Überblick über die gebräuchlichsten Indikatoren, die sich dieser Struktur zuordnen lassen.

Die meisten vorgestellten Indikatoren sind selbsterklärend; „Transferprofil“ und „Drittmittel“" werden an dieser Stelle näher erläutert.

Das Transferprofil oder ,transfer engagement profile“ bezeichnet in der Literatur ganz allgemein die Summe und den Schwerpunkt aller Transferaktivitäten (siehe Rossi und Rosli 2015, S. 1978). Eine Transferstrategie als formalisiertes Transferprofil ist in der Regel in einem Dokument niedergelegt und durch Hochschulgremien legitimiert. Sie zielt darauf ab, Transferprozesse zielorientiert zu befördern; ihre Ausgestaltung wird bedingt durch Typus und Auftrag der Einrichtung, Fächerspektrum und die fachliche Ausrichtung (Wissenschaftsrat 2016, S. 17-18). Das Transferprofil beziehungsweise die Transferstrategie einer Hochschule stellt keinen Input im eigentlichen Sinne dar, beeinflusst allerdings die Bereitstellung bestimmter Ressourcen. In quantitativen Studien wird das Transferprofil zur besseren Vergleichbarkeit herangezogen, um Hochschulen mit ähnlichem Profil vergleichen zu können (siehe Lee und Cho 2014). Der Abgleich der erzeugten Outputs mit festgelegten Zielen einer Transferstrategie gibt darüber hinaus Auskunft über den Erfolg der durchgeführten Transferaktivitäten. Inzwischen dürften wohl die meisten Hochschulen in Deutschland über eine Transferstrategie verfügen.

Das Drittmittelaufkommen lässt sich unter verschiedenen Gesichtspunkten gleich mehreren Indikatorkategorien zuordnen (Woll 2011, S. 49). Zunächst bedeuten Drittmittel Personal- und Sachressourcen, zusätzlich bedeuten eingeworbene Drittmittel auch Reputation (Stifterverband Wissenschaft 2016, S. 8) und spiegeln damit den Erfolg der hochschulischen Transferaktivitäten wider. Im Falle von Drittmittelaufträgen aus der Industrie oder gemeinsamen Forschungsprojekte der Hochschulen mit 
Tab. 1 Übersicht der gebräuchlichsten Indikatoren

\begin{tabular}{ll}
\hline Name Indikator & $\begin{array}{l}\text { Literaturquellen, in denen der Indikator verwen- } \\
\text { det oder bewertet wird }\end{array}$
\end{tabular}
Welchen Teil der
Wirkungskette be-
misst der Indikator

Transferprofil bzw. Transferstrategie (Transfer engagement profile)

Anzahl der Mitarbeiter (Staff)

Akademische

Mitarbeiter/

Verwaltungsmitarbeiter

(Academicl

administrative staff)

Mitarbeiter im Transfer Office (Transfer Office staff)

Drittmittelaufkommen (income from r\&d activities - governmental or private third-party funds)

Anzahl Patentanmeldungen und erteilte Patente (Patent applications/ patents granted)

Veröffentlichte Paper

Netzwerkaktivitäten und Konferenzteilnahmen (networking activities and/or conferences)

Spin-Offs (,IP exploitation", Ausgründung) und Start-ups (,no IP exploitation“, Neugründung)

-

Absolventen (graduates)

Lizenzverträge, Lizenzeinnahmen

Zitationsraten veröffentlichter Paper (citation rates)
Lee and Cho (2014); Rossi and Rosli (2015);

Frank et al. (2019)

Berbegal-Mirabent et al. (2013); Lee und Cho (2014); Thursby und Kemp (2002); Curi et al. (2012)

Berbegal-Mirabent et al. (2013); Curi et al. (2012); Rossi (2018)

Input/Ressource der HEI

Lee and Cho (2014); Siegel et al. (2003); Rossi (2018)

Berbegal-Mirabent et al. (2013); Caldera und Debande (2010); Hewitt-Dundas (2012); Ho et al. (2014); Lee und Cho (2014); Frank et al. (2019); Siegel et al. (2003); Thursby und Kemp (2002)

Agrawal und Henderson (2002); Bozeman (2000); Berbegal-Mirabent et al. (2013); Curi et al. (2012); Ho et al. (2014); Lee und Cho 2014; Rogers et al. (2000); Frank et al. (2019); Thursby und Kemp (2002)

Agrawal und Henderson (2002); Berbegal-Mirabent et al. (2013); Frank et al. (2019)

Agrawal und Henderson (2002); Frank et al. (2019); Wissenschaftsrat (2016)

Input/Ressource der HEI

Input/Ressource der HEI; Output

Output

Output

Output

Berbegal-Mirabent et al. (2013); Caldera und Debande (2010); Lee and Cho (2014); Rogers et al. (2000); Rossi (2018); Frank et al. (2019); Vinig und Lips (2015)

Berbegal-Mirabent et al. (2013); Rossi (2018)

Caldera and Debande (2010); Ho et al. (2014); Lee und Cho (2014); Rogers et al. (2000); Rossi (2018); Frank et al. (2019); Thursby und Kemp (2002); Vinig und Lips (2015)

Agrawal und Henderson (2002); Rossi (2018)
Output Output

Output

Outcome 
Unternehmen kann potentiell auch der Impact auf das Wirtschaftssystem abgebildet werden.

Neben den oben aufgeführten Indikatoren gibt es auch andere mögliche Indikatoren und darüber hinaus existieren bereits viele Ansätze, die Performance hochschulischer Transferaktivitäten systematisch zu evaluieren. In den USA und Kanada erfolgt beispielsweise die jährliche Befragung von Transferstellen durch die Association of University Technology Managers (AUTM). Diese umfasst über 200 Hochschulen und ist hauptsächlich auf die Kommerzialisierung von Technologie fokussiert (Rossi und Rosli 2015, S. 1971), erfasst also eher klassische Aspekte des Technologietransfers. Einige empirische Studien bedienen sich der AUTM Befragung als Datenbasis, wie beispielsweise Ho et al. (2014). Für den europäischen Raum existiert ein vergleichbares Befragungsprogramm der Association of European Science and Technology Transfer Professionals (ASTP), das weitaus differenzierter als die oben aufgeführten Indikatoren ist, aber ähnlich wie die AUTM-Befragung eher auf die Kommerzialisierung von Forschungsergebnissen fokussiert ist. Damit können zwar bestimmte Aspekte des Transfers über einen längeren Zeitraum analysiert und Hochschulen miteinander verglichen werden, eine Beschränkung auf bestimmte Transferaktivitäten führt allerdings dazu, dass Hochschulen mit bestimmten Transferprofilen immer besser abschneiden werden, als andere (siehe auch Rossi und Rosli 2015, S. 1987).

\section{Die Indikatoren im Spiegel der Literatur}

Jenseits des gesicherten positiven empirischen Zusammenhanges zwischen Forschungsausgaben und Wirtschaftswachstum (siehe oben) ist die Messung einer Wirkung von Ausgaben in diesem Bereich auf regionale Wirtschaftssysteme mit großen Schwierigkeiten verbunden, die erhebliche Zweifel wecken, ob die Messung einer Wirkung von Maßnahmen einer Hochschule auf die regionale Wirtschaft überhaupt möglich ist. Im Folgenden sollen nun die ermittelten Indikatoren, die in der gesichteten Literatur besonders häufig benannt wurden, vor dem Hintergrund des Standes der Literatur, der im zweiten Abschnitt referiert wurde, auf ihre Aussagekraft beurteilt werden.

Grundsätzlich gilt für alle Angaben zu Ressourcen, die im Bereich des Technologietransfers verausgabt werden können, insbesondere für die Zahl der Mitarbeiter und die Mittel, die in diesem Bereich verausgabt werden, das Problem des time lag. Ein wirtschaftlicher Effekt des Technologietransfers kann sich erst mit erheblicher Zeitverzögerung einstellen, da jede denkbare Umsetzung ein zeitintensiver Prozess ist. Das gilt für Patente, die wirtschaftlich genutzt werden. Das gilt für Prozessinnovationen, die durch wissenschaftlich getriebene Verfahrensoptimierung herbeigeführt werden. Und das gilt vor allem für die zahlreichen staatlich geförderten Kooperationen zwischen Wirtschaft und Wissenschaft, die zu neuen Produkten führen sollen. In allen diesen Fällen verstreichen im Erfolgsfall Jahre zwischen der Verausgabung von Forschungsmitteln und einer kommerziellen Umsetzung. Auch wenn der Trend zur deutlich schnelleren Vermarktung von Forschungsergebnissen geht, ist der Zeitraum von der Entwicklung einer neuen Idee bis zu ihrer erfolg- 
reichen Umsetzung in den Markt deutlich größer als der Zeitraum der Legitimationszyklen der Akteure, die über die Höhe dieser Ausgaben entscheiden. Innerhalb eines Vierjahreszyklus (die üblichen Wahlzyklen von Landesregierungen, die über Mittel für die Hochschulen entscheiden) oder innerhalb eines Vier- oder Sechsjahreszyklus (Wahlzyklen von Hochschulleitungen) lässt sich deswegen keine Aussage über den wirtschaftlichen Effekt von Transferbemühungen machen. Das gilt mutatis mutandis auch für projektgebunden Ausgaben für den Technologietransfer. Solche Projekte haben i.d.R. eine Zeitspanne von 5 Jahren, in seltenen Fällen auch länger.

Für alle inputbezogenen Indikatoren gilt, dass ihre Wirkung über besonders viele Glieder der kausalen Kette vermittelt ist. Die intermittierenden Faktoren, die auf jeder Stufe dieser Kette eine Rolle spielen, können die Wirkung einer Verbesserung der Ressourcenausstattung von Hochschulen auf die regionale Wirtschaft erheblich beeinträchtigen. Bei allen inputbezogenen Faktoren ist bereits die Eigenlogik des Hochschulsystems in Rechnung zu stellen, die nicht auf die Wirkung auf die regionale Wirtschaft ausgerichtet ist sondern den internen Erfolgskriterien der science community folgt. Hier zählt der Reputationsgewinn durch Veröffentlichung in hoch gerankten wissenschaftlichen Zeitschriften und das Einwerben von Drittmitteln für die Grundlagenforschung bei der DFG mehr als eine denkbare Hilfestellung für einen regionalen Mittelständler.

Eine verbesserte Ressourcenausstattung steht daher in der Gefahr „Zweckentfremdet" zu werden (Kempton 2019), weil sich eine trennscharfe Unterscheidung zwischen Grundlagenforschung und Forschung für den Transfer innerhalb der Hochschulen nur schwer realisieren lässt. Weil eine Verbesserung der Ressourcenausstattung von Hochschulen eine notwenige aber keine hinreichende Bedingung für eine Wirkung auf die regionale Wirtschaft ist, signalisieren Indikatoren, die steigenden Input anzeigen, eher steigende Erwartungen an die Wirkung von Hochschulen auf die regionale Wirtschaft, aber nicht die Wirkung selbst. Für periphere Regionen mit geringer Absorptionsfähigkeit der regionalen Wirtschaft gilt überdies, dass der Anreiz für transferaffine Forscher und Forscherinnen sich finanzstärkere und forschungsaffinere Unternehmen außerhalb der Heimatregion zu suchen, groß ist (Bonnacorsi 2017).

Das Drittmittelaufkommen, insbesondere das Drittmittelaufkommen durch die Industrie wird in der Literatur sowohl als Input als auch als Output qualifiziert. Von einem Inputindikator kann man in diesem Zusammenhang sprechen, weil ein höheres Drittmittelaufkommen signalisiert, dass die Ressourcenausstattung der betreffenden Institution nun eine bessere ist. Von einem Outputindikator kann man in diesem Kontext sprechen, weil die Bewilligung von Fördergeldern für einschlägige Projekte eine spezielle Erwartung der Fördermittelgeber (respektive der gutachtenden Gremien) wiederspiegelt, dass das geförderte Projekt einen spezifischen Erkenntnisgewinn erzeugt, der im Falle von industrienahen Drittmitteln kommerziell nutzbar sein sollte. Deshalb wird das Einwerben von Drittmitteln in den meisten Hochschulen im Rahmen ihrer internen Mittelverteilungsmodelle auch als Leistung bewertet.

Verschiedentlich wird eine Anschärfung dieses Indikators mit Blick auf den Transfer dergestalt vorgenommen, dass insbesondere der Umfang der Drittmittel aus der Industrie oder solche, die für Kooperationsprojekte mit der Industrie eingeworben werden, als Indikator herangezogen werden (siehe Ho et al. 2014, S. 250). 
Es erscheint zunächst plausibel, dass eine größere Nähe zur wirtschaftlichen Verwertung auch mehr Informationsgehalt verspricht. Es liegen jedoch keine belastbaren Studien vor, in welchem Umfang Drittmittel, die nicht von der Industrie eingeworben werden, und Projekte, die nicht mit Partnern der Wirtschaft durchgeführt werden, am Ende doch zu einer wirtschaftlichen Verwertung führen. Angesichts vieler Beispiele, in denen Grundlagenforschung in lukrative Geschäftsmodelle überführt werden konnte, ist deshalb unklar, ob ein Ausschluss solcher Drittmittel bei der Evaluation der Transferleistung einer Hochschule sinnvoll ist.

Auch die Nähe zur Wirtschaft durch Finanzierung oder Projektstruktur ist kein Garant für wirtschaftliche Umsetzung. Ein solches Projekt kann wissenschaftlich scheitern; es kann sich als unwirtschaftlich herausstellen; fehlende Finanzierungsmöglichkeiten können weitere nötige Entwicklungsschritte verunmöglichen usw. usf. Auch hier gilt, industrienahe Drittmittel sind eine notwendige, aber keine hinreichende Bedingung für eine wirtschaftliche Umsetzung. In welchem Umfang industrienahe Forschungsausgaben im Unterschied zur Finanzierung der Grundlagenforschung einen Effekt auf Wachstum oder Produktivität haben, ist ebenfalls nicht geklärt. Dieser Zusammenhang ist nur für Forschungsausgaben insgesamt und ohne Ansehung regionaler Unterschiede empirisch bestätigt (siehe oben).

Bei der Beurteilung des Indikators „Drittmittel“ dürfte erschwerend hinzukommen, dass eine fehlende Entsprechung zwischen den Forschungsschwerpunkten einer Hochschule und den Schwerpunkten der umgebenden regionalen Wirtschaft ebenso wie die fehlende Absorptionsfähigkeit peripherer Wirtschaftsräume dazu führen können, dass ein schwaches Drittmittelaufkommen weniger eine Schwäche dieser Hochschule sondern lediglich eine fehlende Passung zur umgebenden regionalen Wirtschaft signalisiert. Auch der umgekehrte Fall eines hohen Drittmittelaufkommens muss nicht zwangsläufig einen hohen Impact auf die regionale Wirtschaft anzeigen. Denkbar und in peripheren Regionen theoretisch sogar eher zu erwarten ist auch, dass ein erhöhtes Drittmittelaufkommen mit stärkeren Partnern aus der Wirtschaft aus anderen Regionen realisiert wird. Das hohe Drittmittelaufkommen signalisiert in diesem Fall lediglich die Attraktivität der Hochschule für die Wirtschaft im Allgemeinen.

Generell gibt es einen positiven Zusammenhang zwischen der Zahl der Patente eines Landes und seiner wirtschaftlichen Leistungsfähigkeit (Schick 2019). Deswegen gilt die Zahl der Patentanmeldungen als wichtiger Kandidat für einen Indikator, der etwas über den Zusammenhang zwischen Forschungsleistung einer Hochschule einerseits und Wachstum und Produktivität andererseits sagen könnte. Die empirischen Befunde zeigen, dass im deutschen nationalen Innovationssystem in 2018 98,65\% aller Patentanmeldungen durch die Industrie, Privatpersonen oder Forschungseinrichtungen entfielen; auf die Hochschulen hingegen nur 1,35\% (DPMA 2018a, b, eigene Berechnungen). Insbesondere ist in den wirtschaftlich besonders erfolgreichen Bundesländern wie Baden-Württemberg und Bayern das Patentierungsgeschehen durch die Industrie besonders ausgeprägt (DPMA 2018b). Vor diesem Hintergrund dürfte plausibel sein, dass Hochschulen in peripheren Regionen eine schwache Performance des regionalen Innovationssystems kaum durch eine Steigerung der Zahl der Patente, die sie jährlich anmelden, kompensieren können. Dazu ist die Zahl der möglichen Patente im Verhältnis zum Kompensationsbedarf einfach zu gering. 
Als gut gesichert gilt auch, dass auf vergleichsweise wenige Hochschulen der Löwenanteil der Patente entfällt (Henderson et al. 1998; Bulut and Moschini 2006; Rossi 2018, S. 730). Dabei handelt es sich i.d. R. um solche, die eine gründungsaffine regionale Umgebung mit metropolitanem Zuschnitt aufweisen (Bonnacorsi 2017) Die Vermutung liegt nahe, dass die kommerzielle Nutzung von Patenten stark mit der Absorptionsfähigkeit der umgebenden Wirtschaft korrespondiert und es in peripheren Regionen oft an einer solchen innovationsfreundlichen Umgebung mangelt. Die übergroße Mehrheit der Hochschulen weist nur eine geringe Zahl von jährlichen Patenten aus. Kontingente Ereignisse wie die Berufung oder Emeritierung eines einzelnen patentierungsfreudigen Kollegen führen in dieser Gruppe zu Bewegungen dieses Indikators, die wenig über Leistungsfähigkeit dieser Hochschule als Ganzes im Transfer aussagen.

Angesichts der Vielzahl der Patentanmeldungen, denen keine wirtschaftliche Anwendung folgt, ist auch hier die Aussage berechtigt, dass Patente für eine Wirkung auf die regionale Wirtschaft bedeutsam sein können, aber nicht sein müssen. Anders ausgedrückt: Selbst ein hohes Patentaufkommen einer Hochschule kann folgenlos für die umgebende Wirtschaft sein. Die starke Inanspruchnahme von Förderprogrammen in peripheren Regionen, die nicht auf High Tech abzielen sondern lediglich die Zusammenarbeit mit KMU adressieren, spricht für die Vermutung, dass in solchen Regionen der STI-Mode (scientific and technologically-based innovation) unter- und der DUI-Mode (learning-by-doing, by-using, and by-interacting) überrepräsentiert ist (zum Unterschied zwischen diesen beiden Formen der Wissensgenerierung Jensen et al. 2007). Die Annahme, dass im DUI-Mode Patente nicht die entscheidende Rolle spielen, ist ebenfalls plausibel. Eine geringe Zahl von Patentanmeldungen zeigt in solchen Fällen lediglich an, dass Hochschulen keine passenden Partner für ihre Forschungsanstrengungen gefunden haben, bzw. dass die gemeinsamen Projekte mit der regionalen Wirtschaft keine patentierungsfähigen Erkenntnisse abgeworfen haben. Das muss aber nicht ausschließen, dass diese Zusammenarbeit wirtschaftlich bedeutsam war.

Einen deutlichen Schritt weiter geht die Erfassung der Höhe von Lizenzeinnahmen aus Patenten. Solche Einnahmen können nur erwartet werden, wenn das fragliche Patent wirtschaftlich verwertet werden kann oder eine wirtschaftliche Verwertung sehr plausibel erscheint. Wegen der stark ungleichmäßigen Verteilung der Patentierungstätigkeit ist die Annahme schlüssig, dass auch die Einnahmen aus Lizenzen ungleichmäßig verteilt sind. Daher dürfte dieser Indikator für die übergroße Mehrzahl der Hochschulen, bei denen keine oder nur sehr geringe Lizenzeinnahmen anfallen, ebenfalls keinen hohen Informationsgehalt haben, weil auch hier die kleinen Zahlen verhindern, dass die Hochschulen dieser Gruppe untereinander sinnvoll verglichen werden können. Hinzu kommt, dass ebenfalls ein erheblicher Unterschied zwischen Hochschulen in starken regionalen Innovationssystemen und Hochschulen in peripheren Wirtschaftsräumen vermutet werden kann.

Unstrittig ist in der Literatur, dass hochqualifizierte Absolventen, insbesondere solche in den MINT-Fächern als wichtiger Produktionsfaktor erheblich zu Wachstum und Produktivität in der Wirtschaft beitragen können (Schubert et al. 2012, S. 84). Die Wirkung auf die nahräumliche Umgebung einer Hochschule ist hingegen ambivalent, weil zwei gegenläufige Effekte erfasst werden müssen. Einerseits 
qualifiziert eine Hochschule und erschließt damit den Absolventen Arbeitsmärkte jenseits des regionalen Einzugsbereiches einer Hochschule; andererseits kann eine Hochschule durch ihre Ausbildungsleistung das Qualifikationsniveau des regionalen Arbeitsangebotes erhöhen. In peripheren Regionen mit starken demografischen Verlusten, ist nicht a priori klar, welcher dieser Effekte der stärkere ist. Deshalb muss eine hohe Zahl hochqualifizierter Absolventen von Hochschulen in solchen Regionen auch nicht zwangsläufig eine positive Wirkung auf die regionale Wirtschaft signalisieren. Sie kann auch eine Beschleunigung der Abwanderung aus der nahräumlichen Umgebung der Hochschule bedeuten.

Der Output an Papern in referierten Zeitschriften bzw. die Zitierhäufigkeit gilt gemeinhin als guter Indikator für die Leistungsfähigkeit einer Hochschule. Tatsächlich gibt es auch einen nachweislichen Zusammenhang zwischen wissenschaftlicher Leistungsfähigkeit in diesem Sinne und wirtschaftlicher Wirkung. Sehr gute Wissenschaft kann mit wirtschaftlichem Erfolg einhergehen (Bonnacorsi 2017). De facto gestattet das Berichtswesen vieler Hochschulen eine solche aggregierte Erfassung aber nicht, zumeist sind die entsprechenden Leistungen lediglich für einzelne Lehrstühle ausgewiesen. Die wissenschaftlich besonders leistungsfähigen Hochschulen sind überdies meist in Metropolen situiert, wohingegen die Hochschulen, die in peripheren Regionen mit besonderem wirtschaftlichen Entwicklungsbedarf gelegen sind, gemessen an diesen Indikatoren - soweit diese sich erfassen lassen - eher leistungsschwächer sind. (Bonnacorsi 2017 und die dort angegebene Literatur). Folgt man den Hinweisen in der Literatur, scheinen kritische Massen an wissenschaftlicher wie an wirtschaftlicher Leistungsfähigkeit eine wichtige Rolle zu spielen. In peripheren Regionen, wo diese besonders benötigt werden, fehlen sie aber in der Regel auf beiden Seiten der Demarkationslinie zwischen Wissenschaft und Wirtschaft.

Die Zahl der Spin-offs gilt ebenfalls als ein brauchbarer Indikator für den Technologietransfer. Die Geschichte der Technologie- und Gründerzentren, die an vielen Hochschulen angedockt wurden und deren Wirkung ambivalent ist (Phillips 2002, S. 314), zeigt bereits, dass die Existenz einer technisch orientierten Hochschule vielleicht eine notwendige, aber auch keine hinreichende Bedingung für das $\mathrm{Zu}$ standekommen erfolgreicher Ausgründungen ist. Die meisten empirischen Studien stimmen darin überein, dass es zumindest eines gründungsfreundlichen „Eco-Systems“ bedarf, um zahlreiche Gründungen zu ermöglichen. Dazu gehören nicht nur die üblichen Serviceleistungen wie Patentanwälte, sondern auch Firmen, die sich auf die Finanzierung von Gründern spezialisiert haben, ein hochspezialisierter Arbeitsmarkt, auf dem sich auch Fachkräfte für seltene Berufe finden lassen und potentielle Nachfrager in räumlicher Nähe (Acs et al. 2017 und Isenberg 2011) All das findet sich in Metropolen wie Berlin, Hamburg oder München. Das findet sich aber nicht so ohne weiteres in Cottbus, Illmenau oder Zwickau. Hinzu kommt, dass es auch einer Gründungsmentalität bedarf, die in manchen Regionen eine lange Tradition hat, in anderen aber nicht (Fritsch und Wyrwich 2014, S. 956). Eine geringe Zahl von Ausgründungen kann demnach eine schwache Performance der entsprechenden Hochschule anzeigen; das muss aber nicht so sein. Es kann auch einfach bedeuten, dass potentielle Ausgründer aus der Hochschule einen anderen Ort als den ihrer Hochschule als aussichtsreicher angesehen haben oder die Selbstständigkeit kein selbstverständlicher Bestandteil der lokalen Unternehmenskultur ist. 
Kooperationen und Netzwerke zwischen Wirtschaft und Wissenschaft gelten in der Literatur als wichtige Voraussetzung für kooperative Innovationsprozesse (Berger et al. 2018, S. 45; Boschma 2005, S. 65). Eine Messung der Zahl und Dichte dieser Kooperationen ist mit Hilfe von Patentstatistiken, die Aufschluss über die beteiligten Akteure bieten, und mit Hilfe von Förderdatenbanken, die ebenfalls die Kooperationspartner anzeigen, möglich (Berger et al. 2018, S. 66). Auch für Hochschulen lässt sich auf diesem Weg angeben, an wie vielen Netzwerken bzw. Kooperationen sie beteiligt waren oder sind. Umfang und Dichte der Vernetzung stehen zweifellos bereits in einem engen Zusammenhang zu der wirtschaftlichen Verwertung von Inventionen. Als Indikator für die Leistungsfähigkeit von Hochschulen im Transfer sind aber auch diese Angaben nur begrenzt tauglich, weil zu vermuten steht, dass sie stark mit der Absorptionsfähigkeit des regionalen Innovationssystems korrelieren. Wenn eine Hochschule am Stadtrand von Berlin also mehr solcher Kooperationen ausweist als eine an der Peripherie Brandenburgs, dann darf man vermuten, dass das regionale Innovationssystem im Speckgürtel von Berlin eine höhere Absorptionskapazität hat als das regionale Innovationssystem in den Randlagen Brandenburgs. Ob mit solchen Befunden eine vergleichende Aussage über die Leistungsfähigkeit des Transfers beider Hochschulen begründet werden kann, ist hingegen zumindest zweifelhaft.

\section{Zusammenfassung und Ausblick}

Eine Aussage, wonach die Leistungsfähigkeit einer Hochschule im Transfer der entscheidende Faktor des wirtschaftlichen Aufschwungs in einer Region ist, ist auf der Basis der diskutierten Indikatoren nicht ohne weiteres möglich. Dem stehen der Zeithorizont solcher Entwicklungen und die Vielzahl intermittierender Faktoren entgegen. Das schließt nicht aus, dass einer Hochschule regionale wirtschaftliche Entwicklungen zugerechnet werden können. Wenn zum Beispiel ein Unternehmen sich in der Nähe einer Hochschule ansiedelt, weil in der Hochschule ein Lehrstuhlinhaber Entwicklungen betreibt, von denen das Unternehmen profitieren kann, dann ist das zweifelsfrei ebenso ein Beitrag der Hochschule für die regionale wirtschaftliche Entwicklung wie die Einstellung eines Absolventen in einem Unternehmen der Region.

Möglich sind hingegen Aussagen derart, dass Hochschulen ihren wirtschaftsbezogenen Output steigern oder dass dieser sinkt. So kann zum Beispiel die Anzahl der Patente, die eine Hochschule anmeldet, steigen oder sinken; die Hochschule kann sich an mehr oder weniger Netzwerken mit Wirtschaftsbeteiligung beteiligen; das Drittmittelaufkommen aus Industrieaufträgen kann größer oder kleiner werden; die Zahl der Ausgründungen mehr oder weniger etc.

Aussagekräftig sind auch Indikatoren, die als Produktivitätsindikatoren angesehen werden können. So könnte man zum Beispiel fragen, wie hoch das Drittmittelaufkommen aus der Industrie im Verhältnis zur Größe der Hochschule ist (gemessen durch den Umfang des Globalhaushaltes oder die Zahl der Mitarbeiter). Solche Indikatoren sagen etwas über die Effizienz der Mittelverwendung mit Blick auf den Output einer Hochschule, setzen aber voraus, dass der Output dieser Hochschule in 
einem sinnvollen Zusammenhang zur wirtschaftlichen Entwicklung der Umgebung steht.

Ob und in welchem Umfang die Steigerung dieses Outputs dann zum Wachstum der regionalen Wirtschaft bzw. deren Produktivität beiträgt, hängt zu einem erheblichen Teil von Faktoren ab, auf die eine Hochschule wenig oder gar keinen Einfluss hat (Struktur und Absorptionsfähigkeit der regionalen Wirtschaft, die Existenz einer ausgründungsfreundlichen Umgebung, etc.). Ein Urteil über den Erfolg oder Impact des Transfers einer Hochschule in diesem Sinne ist deshalb solange nicht sinnvoll, wie der Einfluss dieser intermittierenden Faktoren auf die Ausprägungen, die die Indikatoren annehmen können, nicht in der Statistik neutralisiert werden kann.

Hinzu kommt, dass TT (wie auch die Mobilität der Absolventen als Humankapital für den Arbeitsmarkt) nicht ausschließlich intraregional, sondern oft interregional stattfindet, insbesondere bei Hochschulen mit hohem Prestige bzw. Firmen mit hoher Absorptionsfähigkeit. Das impliziert einen Zielkonflikt zwischen der Stärkung der Region und der Stärkung des eigenen wissenschaftlichen Outputs und internationalen Prestiges einer Hochschule sowie die Schwierigkeit der Trennung von regionalen und nationalen oder gar globalen Impacts des WTT.

Erfolgreicher Technologietransfer hat einen Metropolenbias. Weil Indikatoren aus diesem Grund oft nur anzeigen, dass sich der TT in einer metropolitanen Region erfolgreicher gestalten lässt als in einer peripheren, sind Vergleiche zwischen Hochschulen in Metropolen und solchen in peripheren Wirtschaftsräumen nur beschränkt sinnvoll.

Aus diesen Ergebnissen folgt nicht, dass die Hochschulen nicht dazu angehalten werden sollten, intensiv TT zu betreiben. Das bedeutet lediglich, dass man sich von der Vorstellung verabschieden muss, dass der Einfluss solcher Bemühungen auf die regionale Wirtschaft mit Händen (oder Indikatoren) zu greifen ist. Die diskutierten Indikatoren sagen etwas über Outputs der Hochschule; ob und in welchem Umfang diese dann die regionale Wirtschaft stimulieren, steht auf einem anderen Blatt.

Danksagung Die Autoren danken dem Bundesministerium für Bildung und Forschung für die finanzielle Unterstützung im Rahmen der Förderinitiative „Innovative Hochschule“ und des Projektes „Innovation Hub 13 - fast track to transfer“.

Funding Open Access funding provided by Projekt DEAL.

Open Access Dieser Artikel wird unter der Creative Commons Namensnennung 4.0 International Lizenz veröffentlicht, welche die Nutzung, Vervielfältigung, Bearbeitung, Verbreitung und Wiedergabe in jeglichem Medium und Format erlaubt, sofern Sie den/die ursprünglichen Autor(en) und die Quelle ordnungsgemäß nennen, einen Link zur Creative Commons Lizenz beifügen und angeben, ob Änderungen vorgenommen wurden.

Die in diesem Artikel enthaltenen Bilder und sonstiges Drittmaterial unterliegen ebenfalls der genannten Creative Commons Lizenz, sofern sich aus der Abbildungslegende nichts anderes ergibt. Sofern das betreffende Material nicht unter der genannten Creative Commons Lizenz steht und die betreffende Handlung nicht nach gesetzlichen Vorschriften erlaubt ist, ist für die oben aufgeführten Weiterverwendungen des Materials die Einwilligung des jeweiligen Rechteinhabers einzuholen.

Weitere Details zur Lizenz entnehmen Sie bitte der Lizenzinformation auf http://creativecommons.org/ licenses/by/4.0/deed.de. 


\section{Literatur}

Acs, Z. J., Stam, E., Audretsch, D. B., \& O'Connor, A. (2017). The lineages of the entrepreneurial ecosystem approach. Small Business Economics, 49(1), 1-10.

Aghion, P., \& Howitt, P. (2006). Appropriate growth policy: a unifying framework. Journal of the European Economic Association, 4(2/3), 269-314.

Aghion, P., Ljungqvist, L., Howitt, P., Howitt, P.W., Brant-Collett, M., \& García-Peñalosa, C. (1998). Endogenous growth theory. Cambridge: MIT Press.

Agrawal, A., \& Henderson, R. (2002). Putting patents in context: exploring knowledge transfer from MIT. Management Science, 48(1), 44-60. https://doi.org/10.1287/mnsc.48.1.44.14279.

Berbegal-Mirabent, J., Lafuente, E., \& Solé, F. (2013). The pursuit of knowledge transfer activities: an efficiency analysis of Spanish universities. Journal of Business Research, 66(10), 2051-2059. https:// doi.org/10.1016/j.jbusres.2013.02.031.

Berger, W., Lange, R., Schnellenbach, J., \& Titze, M. (2018). Empirische Analyse und Konzepterarbeitung „Entrepreneurship-Indikator Lausitz“: Ein Ansatz. Gutachten für die Innovationsregion. Halle (Saale) und Cottbus: Innovationsregion Lausitz GmbH.

Bonaccorsi, A. (2017). Addressing the disenchantment: universities and regional development in peripheral regions. Journal of Economic Policy Reform, 20(4), 293-320.

Bonaccorsi, A., Daraio, C., \& Simar, L. (2006). Advanced indicators of productivity of universities. An application of robust nonparametric methods to Italian data. Scientometrics, 66(2), 389-410.

Boschma, R. (2005). Proximity and innovation: a critical assessment. Regional Studies, 39(1), 61-74. https://doi.org/10.1080/0034340052000320887.

Boschma, R., \& Gianelle, C. (2014). Regional branching and smart specialisation policy. JRC88242. (S. 741-765). Luxembourg: Institute for Prospective and Technological Studies, Joint Research Centre.

Bozeman, B. (2000). Technology transfer and public policy: a review of research and theory. Research Policy, 29(4-5), 627-655. https://doi.org/10.1016/S0048-7333(99)00093-1.

Brachert, M., Dettmann, E.,Titze, M. (2019) The regional effects of a place-based policy - Causal evidence from Germany. Regional Science and Urban Economics, 79:103483.

Bulut, H., \& Moschini, G. (2006) Patents, trade secrets and the correlation among R\&D projects. Economics Letters, 91(1), 131-137.

Bundesministerium für Bildung und Forschung (BMBF) (2018). Forschung und Innovation für die Menschen: Die Hightech-Strategie 2025. Berlin: Bundesministerium für Bildung und Forschung (BMBF).

Caldera, A., \& Debande, O. (2010). Performance of Spanish universities in technology transfer: An empirical analysis. Research Policy, 39(9), 1160-1173. https://doi.org/10.1016/j.respol.2010.05.016.

Cohen, W. M., \& Levinthal, D. A. (1990). Absorptive capacity: a new perspective on learning and innovation. Administrative science quarterly, 35(1), 128-152. https://doi.org/10.2307/2393553

Cooke, P. (2001). Regional innovation systems, clusters, and the knowledge economy. Industrial and corporate change, 10(4), 945-974.

Curi, C., Daraio, C. C., \& Llerena, P. (2012). University technology transfer: how (in-)efficient are French universities? SSRN Journal. https://doi.org/10.2139/ssrn.1984043.

Deutsches Patent- und Markenamt (2018a). Patentanmeldungen der Hochschulen nach Bundesländern. CSV-Statistiken. https://www.dpma.de/docs/dpma/veroeffentlichungen/statistik/csv2018/ patentanmeldungenderhochschulennachbundeslaendern2014bis2018.csv. Zugegriffen: 24. Apr. 2020.

Deutsches Patent- und Markenamt (2018b). Patentanmeldungen nach Bundesländern. CSV-Statistiken. https://www.dpma.de/docs/dpma/veroeffentlichungen/statistik/csv2018/patentanmeldungeninland dpmanachbundeslaendern.csv. Zugegriffen: 24. Apr. 2020.

Etzkowitz, H. (1997). The entrepreneurial university and the emergence of democratic corporatism. In Universities and the global knowledge economy: a triple helix of university-industry-government relations (S. 141-152). London: Pinter.

Etzkowitz, H. (2008). The triple helix. University-industry-government innovation in action. New York: Routledge. Online verfügbar unter http://site.ebrary.com/lib/alltitles/docDetail.action?docID= 10227367

European Union (2011). Connecting universities to regional growth: a practical guide

Faggian, A., \& McCann, P. (2008). Human capital, graduate migration and innovation in British regions. Cambridge Journal of Economics, 33(2), 317-333. https://doi.org/10.1093/cje/ben042. 
Frank, A., Heinlein, M., Lehmann-Brauns, C., Lohr, F., Schröder, C., \& Schröder-Kralemann, A.K. (2019). Erfolgsmessung von Transfer und Kooperation an Hochschulen. Diskussionspapier, 2019 Ausgabe 2. Essen: Stiftverband für Deutsche Wissenschaft e.V.. Online verfügbar unter https://www.stifterverband.org/sites/default/files/erfolgsmessung_von_transfer_und_kooperation_ an_hochschulen.pdf

Fritsch, M., \& Wyrwich, M. (2014). The long persistence of regional levels of entrepreneurship: Germany, 1925-2005. Regional Studies, 48(6), 955-973.

Henderson, R., Jaffe, A., Trajtenberg, M. (1998) Universities as a Source of Commercial Technology: A Detailed Analysis of University Patenting, 1965-1988. Review of Economics and Statistics, 80(1), 119-127.

Hewitt-Dundas, N. (2012). Research intensity and knowledge transfer activity in UK universities. Research Policy, 41(2), 262-275. https://doi.org/10.1016/j.respol.2011.10.010.

Ho, M.H.-C., Liu, J.S., Lu, W.-M., \& Huang, C.-C. (2014). A new perspective to explore the technology transfer efficiencies in US universities. The Journal of Technology Transfer, 39(2), 247-275. https:// doi.org/10.1007/s10961-013-9298-7.

Isenberg, D. (2011). The entrepreneurship ecosystem strategy as a new paradigm for economy policy: principles for cultivating entrepreneurship. Babson Park: Babson Entrepreneurship Ecosystem Project, Babson College.

Jensen, M. B., Johnson, B., Lorenz, E., \& Lundvall, B.-Å. (2007). Forms of knowledge and modes of innovation. In B. A. Lundvall (Hrsg.), The learning economy and the economics of hope (S. 155). London: Anthem.

Kempton, L. (2019). Wishful thinking? Towards a more realistic role for universities in regional innovation policy. European Planning Studies, 27(11), 2248-2265.

Kritikos, A. S., Hafenstein, M., \& Schiersch, A. (2017). Auch kleinste Betriebe stoßen erfolgreich Innovationen an, sie tun es nur seltener. DIW-Wochenbericht, 84(37), 755-761.

Lee, S.S., \& Cho, J.M. (2014). The measurement and evaluation of technology transfer activities: indicators for university and research institution. The Journal of Intellectual Property, 9(1), 157-179.

Ministerium für Wissenschaft, Forschung und Kultur des Landes Brandenburg (2019a). Strukturwandel erfolgreich gestalten - Lausitz-Strategie des Ministeriums für Wissenschaft, Forschung und Kultur. Ministerium für Wissenschaft, Forschung und Kultur des Landes Brandenburg. Potsdam. https://mwfk. brandenburg.de/sixcms/media.php/9/Lausitzstrategie_MWFK.pdf. Zugegriffen: 14. März 2020.

Ministerium für Wissenschaft, Forschung und Kultur des Landes Brandenburg (2019b). Umsetzungsplan für die Lausitz-Strategie des Ministeriums für Wissenschaft, Forschung und Kultur. Ministerium für Wissenschaft, Forschung und Kultur des Landes Brandenburg. Potsdam. https://mwfk.brandenburg. de/sixcms/media.php/9/Umsetzungsplan.pdf. Zugegriffen: 14. März 2020.

Phillips, R.G. (2002). Technology business incubators: how effective as technology transfer mechanisms? Technology in Society, 24(3), 299-316. https://doi.org/10.1016/S0160-791X(02)00010-6.

Rassenhövel, S. (2010). Performancemessung im Hochschulbereich. Wiesbaden: Gabler.

Research Councils UK (2007). Knowledge Transfer Categorisation and Harmonisation Project. Final Report. Swindon: Research Councils UK.

Rogers, E. M., Yin, J., \& Hoffmann, J. (2000). Assessing the effectiveness of technology transfer offices at US research universities. The Journal of the Association of University Technology Managers, 12, 47-80

Rossi, F. (2018). The drivers of efficient knowledge transfer performance: evidence from British universities. Cambridge Journal of Economics, 42(3), 729-755. https://doi.org/10.1093/cje/bex054.

Rossi, F., \& Rosli, A. (2015). Indicators of university-industry knowledge transfer performance and their implications for universities: evidence from the United Kingdom. Studies in Higher Education, 40(10), 1970-1991. https://doi.org/10.1080/03075079.2014.914914.

Schick, I.C. (2019). The relationship between annual patent filings and GDP. https://www.legalbusiness world.com/single-post/2019/04/11/The-Relationship-Between-Annual-Patent-Filings-and-GDP. Zugegriffen: 6. Mai 2020. Legal Business World Publications, 11.04.2019.

Schmidt, J. (2013). Ansätze zur Leistungserfassung im Wissens- und Technologietransfer aus Hochschulen in Deutschland. Gesellschaftliche Anforderungen, hochschulspezifische Rahmenbedingungen und mögliche Evaluationskonzepte. Schriftenreihe Lehre \& Forschung, Bd. 17. Hamburg: Kovač. Zugl.: Lüneburg, Univ., Diss., 2012

Schubert, T., Baier, E., Hufnagl, M., Meyer, N., Schricke, E., \& Stahlecker, T. (2012). Endbericht zur Metastudie Wirtschaftsfaktor Hochschule. Stifterverband für die Deutsche Wissenschaft. Karlsruhe: Fraunhofer-Institut für System-und Innovationsforschung ISI. 
Schultz, E. (2013). Wissenstransfer in die Mediengesellschaft. Situationsanalyse und Orientierungshilfen. Beiträge zur Hochschulpolitik, 2013,3. Bonn: HRK Hochschulrektorenkonferenz. Online verfügbar unter http://www.hrk.de/fileadmin/redaktion/hrk/02-Dokumente/02-10-Publikationsdatenbank/ Beitr-2013-03_Wissenstransfer.pdf

Siegel, D. S., Waldman, D., \& Link, A. (2003). Assessing the impact of organizational practices on the relative productivity of university technology transfer offices: an exploratory study. Research Policy, 32(1), 27-48. https://doi.org/10.1016/S0048-7333(01)00196-2.

Stam, E. (2015). Entrepreneurial ecosystems and regional policy: a sympathetic critique. European Planning Studies, 23(9), 1759-1769.

Statistisches Bundesamt (Destatis) (2018). Hochschulen auf einen Blick. Unter Mitarbeit von Juliane Gude und Alexander Schacht (Ausgabe 2018). https://www.destatis.de/DE/Themen/Gesellschaft-Umwelt/ Bildung-Forschung-Kultur/Hochschulen/Publikationen/Downloads-Hochschulen/broschuere-hoch schulen-blick-0110010187004.pdf?_blob=publicationFile. Zugegriffen: 23. April 2020.

Teixeira, A. A. C., Oliveira, A., Daniel, A. D., Preto, M. T., Brás, G. R., \& Rodrigues, C. (2020). The Impact of Universities on Regional Competitiveness: A Review of the Main Theoretical and Methodological Approaches. In Examining the Role of Entrepreneurial Universities in Regional Development (S. 67-92). Hershey: IGI Global.

Thursby, J., \& Kemp, S. (2002). Growth and productive efficiency of university intellectual property licensing. Research Policy, 31(1), 109-124.

Valero, A., \& van Reenen, J. (2019). The economic impact of universities: evidence from across the globe. Economics of Education Review, 68, 53-67. https://doi.org/10.1016/j.econedurev.2018.09.001.

Vinig, T., \& Lips, D. (2015). Measuring the performance of university technology transfer using meta data approach: the case of Dutch universities. The Journal of Technology Transfer, 40(6), 1034-1049. https://doi.org/10.1007/s10961-014-9389-0.

Wissenschaftsrat (2016). Wissens- und Technologietransfer als Gegenstand institutioneller Strategien. Positionspapier. Hg. v. Wissenschaftsrat (Drs. 5665-16). https://www.wissenschaftsrat.de/download/ archiv/5665-16.pdf. Zugegriffen: 15. Aug. 2018.

Woll, C. (2011). Wie lassen sich Forschungsleistungen messen? Entwicklung eines Indikatorensets zur Anwendung aufdem Gebiet der Berufsbildungsforschung. Wissenschaftliche Diskussionspapiere, Bd. 131. Bonn: Bundesinstitut für Berufsbildung.

Zou, T., Ertug, G., \& George, G. (2018). The capacity to innovate: a meta-analysis of absorptive capacity. Innovation, 20(2), 87-121. https://doi.org/10.1080/14479338.2018.1428105. 